\title{
Experimental Outlook for the Pentaquark
}

\author{
Ken Hicks * \\ Department of Physics and Astronomy, Ohio University, \\ Athens, Ohio 45701, USA
}

June 17, 2018

\begin{abstract}
A critical look is taken at both positive and null evidence for the $\Theta^{+}$ pentaquark. Potential problems with experiments will be discussed and the question of what conclusion can be drawn from both the positive and the null results is examined. First the question of existence of the $\Theta^{+}$ pentaquark is considered, followed by a discussion of new experiments that are either planned or in progress to answer questions about its mass, width and isospin. Finally, indirect evidence for the parity of the $\Theta^{+}$is examined, and suggestions for experiments to measure its parity directly are given.
\end{abstract}

\section{$1 \quad$ Introduction}

The possible existence of the $\Theta^{+}$remains one of the most exciting topics in nuclear physics. To date there are over ten experiments 1 with evidence for this state, and a similar number of high-energy experiments 2] that see no evidence for the $\Theta^{+}$even though other states, such as the $\Lambda(1520)$ hyperon resonance, are seen clearly. Furthermore, the $\Theta^{+}$is not seen in $e^{+} e^{-}$collisions 3 where, for example, the $\mathrm{J} / \psi$ or the $\psi(2 s)$ could decay to a $\Theta^{+}$and anti- $\Theta^{+}$final state. On the other hand, in the latter two cases we can only guess at the $\Theta^{+}$production mechanism (or lack thereof). In contrast, several experiments at medium energies have exclusive final states and production cross sections that can be calculated 4] within a theoretical model, given some reasonable assumptions.

Can the contrasting results of these experiments be understood? This is the central theme of the present paper, along with an overview of experimental results expected in the near future. If the $\Theta^{+}$exists, it is important to design experiments that can accurately measure its width, spin and parity. First, a critical look is taken at both positive and null experimental results for the $\Theta^{+}$ pentaquark.

\footnotetext{
*email: hicks@ohio.edu
} 


\section{Does the $\Theta^{+}$Exist?}

Virtually all experiments are subject to some criticism. It is quite difficult to understand the systematic uncertainties in a measurement, and this is especially true when the statistics are limited. The experiments with evidence for the $\Theta^{+}$ have low statistics, and the background under the peaks may not be completely understood. As a result, the statistical significance of the evidence has been questioned. It makes sense to focus on the most reliable experiments to answer the question of whether the $\Theta^{+}$exists.

The first experiments from the LEPS [5], DIANA [6], CLAS [7] and SAPHIR 8 collaborations were ground-breaking, but each has some weakness. The LEPS experiment had only 19 counts in the peak on top of a background that was 17 counts, so detailed studies of the systematics of the background and the Fermi motion correction were difficult. (New data from LEPS with more statistics will be presented below.) The DIANA experiment is hampered by background from kaon charge-exchange reactions, and not enough detail is given in their paper to show how the cuts they employ to reduce this background affect the mass spectrum with the $\Theta^{+}$peak, which is concentrated into a single bin. The CLAS data was the first exclusive reaction on the $\Theta^{+}$but requires a complicated mechanism with secondary-scattering to give energy to the proton, which would otherwise be a spectator. As a result, the shape of the background under the $\Theta^{+}$peak is difficult to estimate and may include kinematic reflections 9 . The SAPHIR collaboration was the first to publish for the $\gamma p \rightarrow K_{s}^{0} K^{+} n$ reaction, but the large cross section they estimated from their measurement conflicted with data for the same reaction from CLAS 10. A re-analysis of the SAPHIR data 11] suggests a smaller cross section but is still under study.

Following the first reports, several experiments measured the invariant mass of the $K_{s}^{0}$ and a proton, which showed a peak close to the $\Theta^{+}$mass, from inclusive production. One of these collected data from neutrino experiments (ITEP [12]) and two others used electroproduction (HERMES 13] and ZEUS [14]). Of course, the $K_{s}^{0}$ is a mixture of both strangeness +1 and -1 , so the invariant mass spectra will include both $\Sigma^{*+}$ and possible $\Theta^{+}$peaks. It follows that a peak at a mass where no $\Sigma^{*+}$ resonance is known could be evidence for the $\Theta^{+}$ or an unknown $\Sigma^{*+}$ resonance. It is also curious that these three measurements reported a $\Theta^{+}$mass which is about $10 \mathrm{MeV}$ below that seen by the first experiments (barely compatible within the experimental uncertainties). Furthermore, most of the null evidence for the $\Theta^{+}$(see below) also measure the $p K_{s}^{0}$ invariant mass, but no peak is seen at the $\Theta^{+}$mass. The inherent weakness in not knowing the strangeness of a particle, coupled with the uncertainty in the background which must include the overlapping $\Sigma^{*+}$ resonances, makes this evidence less convincing than exclusive measurements.

Three experiments remain that have good evidence for the $\Theta^{+}$. The first is from CLAS on a proton target [15. More details will be given separately 16 but a few comments are in order. This exclusive reaction, $\gamma p \rightarrow \pi^{+} K^{-} K^{+} n$ is very clean, and the background comes primarily from meson production reactions. The cuts for this analysis were not chosen arbitrary, as has been suggested by 


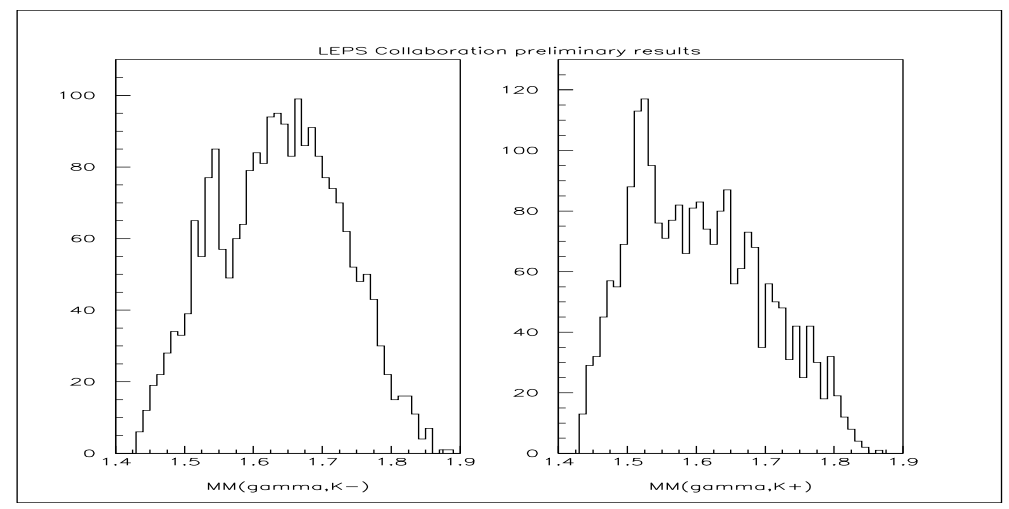

Figure 1: Preliminary missing mass spectra for the $\gamma d \rightarrow K^{+} K^{-} X$ reaction measured with the LEPS detector at SPring- 8 . The event selection requires: (1) particle ID for each kaon; (2) the missing mass of the $K K$ system is within $5 \mathrm{MeV}$ of the nucleon mass; (3) a cut on the $K K$ invariant mass to remove the $\phi$-meson resonance; (4) the photon energy is less than $2.35 \mathrm{GeV}$. The $\Theta^{+}$peak is seen at about $1.53 \mathrm{GeV}$ on the left and the $\Lambda(1520)$ peak is seen at $1.52 \mathrm{GeV}$ on the right.

some critics, but are specifically designed to remove the dominant background along with the assumption that the $\Theta^{+}$can be produced through an s-channel diagram [15. Furthermore, these data were examined by a partial wave analysis (PWA), where the amplitudes of each partial wave were fit over the full angular coverage of the CLAS detector. Hence, the background under the $\Theta^{+}$peak (after all cuts are applied) has been fixed by the PWA from the full (uncut) data. The $\Theta^{+}$peak here has the highest statistical significance yet reported, in excess of $7 \sigma$. Because this is an exclusive measurement from the proton, there is no ambiguity in rescattering from other nucleons, and the strangeness of the final state is clearly identified. On the other hand, the mass of the peak is at $1.55 \pm 0.01 \mathrm{GeV}$, which is about $0.01 \mathrm{GeV}$ higher than the initial $\Theta^{+}$ measurements.

The second experiment with good evidence for the $\Theta^{+}$is the COSY-TOF result from the exclusive hadronic reaction $p p \rightarrow \Sigma^{+} K_{s}^{0} p$. Here, the strangeness of the $p K_{s}^{0}$ invariant mass is tagged by the $\Sigma^{+}$. The particle identification is done entirely by geometric reconstruction which, for this near-threshold reaction, is shown to be very accurate. The details are given separately in these proceedings [17. Some critics have questioned whether this method provides good identification of the final state, but it can be rigorously proven that the kinematics are over-constrained 17. The result is a very clean final state showing a $\Theta^{+}$peak at a mass of about $1.53 \mathrm{GeV}$, which is on the low side of the $\Theta^{+}$ mass measurements.

The third experiment, and perhaps the most convincing one, is the new data 
from LEPS on a deuterium target. These data are shown in Fig. 1, which uses minimal cuts for the event selection. There are clear peaks for both the $\Lambda(1520)$ and the $\Theta^{+}$where the same event sample has been used for both plots and the same Fermi motion correction is applied to both. The only difference is that one spectrum uses the $K^{+}$and the other uses the $K^{-}$, and the detector acceptance is symmetric for these charged kaons. In addition, the same analysis procedures applied to a mixed-event test do not show any peaks at the location of either the $\Theta^{+}$or the $\Lambda(1520)$. In the mixed-event test, the $K^{+}$and $K^{-}$are taken from different events, but the same analysis cuts (which ensure energy and momentum conservation) are applied. Further confidence is gained by seeing that the peaks cannot be generated from a kinematic reflection of $K^{+} K^{-}$pairs from the $\phi$-meson peak. More details are given in a talk by Nakano 18 .

\section{$2.1 \quad$ Null Results}

Having taken a critical look at the evidence in favor of the $\Theta^{+}$we now turn to the null results. These have all come from either high-energy reactions using a hadron beam (such as HERA-B, HyperCP, and CDF 19]) or from electronpositron colliders (Belle, BaBar, BES [3] $)$. Because of the difficulty in detecting neutrons in these detectors, typically these experiments look at the $p K_{s}^{0}$ invariant mass, like in the HERMES and ZEUS experiments. Unlike the mediumenergy electroproduction reactions, the high-energy hadron beam experiments typically have much higher statistics yet see no $\Theta^{+}$peak. Naively, one might expect that if the $\Theta^{+}$exists, it should be produced in both high-energy electroproduction and high-energy hadron collisions, perhaps through fragmentation processes as the flux tube breaks when the struck quark exits the nucleon. This reasoning suggests that the $\Theta^{+}$does not exist. On the other hand, for the electron-positron collisions, it is not clear how an appreciable number of $\Theta-\bar{\Theta}$ pairs are produced by a mechanism where 5 quarks and 5 antiquarks must be produced from, say, decay of a $q \bar{q}$ meson.

Since the hadron beam experiments pose a more serious challenge to the existence of the $\Theta^{+}$we should examine these experiments with some care. In the interest of fairness, the same criticism directed at the HERMES and ZEUS experiments should also be applied to the high-energy hadron experiments. Perhaps the most severe criticism is that the $p K_{s}^{0}$ spectra should show evidence for known $\Sigma^{*+}$ resonances, even if these resonances are broad, yet these spectra are featureless even with high statistics. Clearly, more effort needs to be put toward understanding the background in these measurements.

The production mechanism of the $\Theta^{+}$(if it exists) or even the $\Lambda^{*}$ and $\Sigma^{*}$ resonances from fragmentation processes is not well known. Hence the highenergy experiments can only put an upper limit on the ratio of production of, say, the pair of $\Sigma^{*}$ resonances at $1660-1670 \mathrm{MeV}$ to $\Lambda(1520)$, or $\Theta^{+}$to $\Lambda(1520)$. Non-observation of the $\Sigma^{*}$ resonances, which might be difficult to detect because of their broad width, does not mean that the $\Sigma^{*}$ does not exist. Similar reasoning applies to the non-observation of a $\Theta^{+}$peak, although the limits will be more stringent because of its narrow width. When examining the results from high- 
energy hadron beams, it is important to report not just the upper limit on $\Theta^{+}$ but also the upper limits on other known hadron resonance production. These upper limits should then be confronted quantitatively with calculations based on models of flux-tube fragmentation.

Finally, the facts should be clearly stated when drawing conclusions from both positive and null evidence. The kinematics in experiments with upper limits on $\Theta^{+}$production are different from those experiments reporting positive evidence. In other words, the null results do not prove that the positive results are wrong. There may be some interesting physics to be learned, assuming the experiments are correct, as to why exclusive measurements at medium energy show a potential $\Theta^{+}$peak whereas this signal seems to be obscured in high-energy inclusive measurements. Regardless of the explanation, the facts (and assumptions) should be made clear when drawing conclusions about the existence of the $\Theta^{+}$pentaquark.

\section{$2.2 \quad$ Experimental outlook}

Two measurements expected to produce about a ten-fold increase in statistics, one using a deuterium target and the other using a hydrogen target, are currently being analyzed from CLAS. However results are not expected to be ready until late 2004. In addition, another experiment to get more statistics from the COSY-TOF detector is planned within the year. High statistics will be a crucial test of whether the $\Theta^{+}$exists or not.

Analysis of the new deuterium data from CLAS is in progress. The missing mass spectrum for the $\gamma p \rightarrow K^{+} K^{-} p n$ reaction, where only the charged particles are detected, shows a clean neutron peak with very litle background. The photon beam had a maximum energy of $3.6 \mathrm{GeV}$, compared with the published data [7] which was taken at two photon beams with 2.3 and $3.0 \mathrm{GeV}$ maximum energy. The higher beam energy and the lower magnetic field of the new measurement provide different kinematics than before 7 . (Note that one can match the kinematics of the earlier CLAS data by limiting the photon energy and making angular cuts in the data analysis.) Because of the importantce of "getting it right the first time", the CLAS collaboration has chosen not to release this mass spectrum for the $n K^{+}$system (where the $\Theta^{+}$peak would be expected) until the analysis results on the full data set are final.

Similar comments apply to the new proton data from CLAS. However, in this case the photon beam energy is lower than the published result [15], which had the majority of the data taken at a maximum photon energy of $5.4 \mathrm{GeV}$. The new data are focussed on a measurement of the reaction $\gamma p \rightarrow K^{0} K^{+} n$, similar to that reported by the SAPHIR collaboration 8 . Preliminary data on this reaction was reported at the NSTAR 2004 conference 20 but these data have not been published because higher statistics were desired. Indeed, the new data will provide at least a ten-fold increase in statistics in the desired photon energy range.

The measurement at COSY-TOF will take data with improved detector resolution within the next year, and could increase the statistics of the reported 
results 21 by as much as a factor of five. In addition, the new data are planned at a slightly higher beam energy so that the detector acceptance is more uniform in the region above the $\Theta^{+}$peak. Again, this measurement could be definitive if the reported results are reproduced with higher statistics. On the other hand, if the peak is not reproduced then it will be important to find an explanation of the previous COSY-TOF $\Theta^{+}$peak.

\section{How small is the $\Theta^{+}$width?}

One of the most curious features of the $\Theta^{+}$is its apparent narrow width. Because the $\Theta^{+}$can "fall apart" (without quark pair creation) into a kaon and a nucleon, its width is expected to be hundreds of $\mathrm{MeV} 22,23,24$. On the other hand, the chiral soliton model predicts a narrow width 25 for the $\Theta^{+}$based on symmetries of the model (along with known constants such as the pion decay constant). However, the width predicted by the chiral solition model has significant model dependence 26, 27] and so may not be sufficient by itself to explain a width as narrow as $1.0 \mathrm{MeV}$ 27]. The quark model can also obtain a width on the order of $5-10 \mathrm{MeV}$ if there are correlations between quarks, such as diquark pairs in the $\Theta^{+}$wavefunction 23, 24, 28]. If the $\Theta^{+}$exists and has a width of a few $\mathrm{MeV}$ or less, then this presents a significant challenge to the conventional quark model.

Unfortunately, only upper limits on the $\Theta^{+}$width are available from the experiments described above. The smallest upper limit comes from the DIANA collaboration result, which shows a peak width of $9 \mathrm{MeV}$. However, Cahn and Trilling 30 have shown that the DIANA result implies an intrinsic resolution of the $\Theta^{+}$on the order of $1 \mathrm{MeV}$. Other studies of the phase-shifts determined from older $K N$ scattering data 29, 31] suggest that if the $\Theta^{+}$exists with a mass in the range of 1530 to $1550 \mathrm{MeV}$, then its width is likely to be about $1 \mathrm{MeV}$ or less. More recent preprints 32, 33] have done careful calculations which compare directly with the $K N$ data and come to a similar conclusion that the $\Theta^{+}$width is on the order of $1 \mathrm{MeV}$. On the other hand, the $K N$ database is very sparse in this energy range, corresponding to the $\Theta^{+}$mass, and better data is desired 34.

Two experiments have measured a $\Theta^{+}$width that is not just an upper limit. The HERMES experiment 13 has quoted a width of $13 \pm 9 \pm 3 \mathrm{MeV}$, where the first uncertainty is statistical and the second one is systematic, which is larger than the experimental resolution of 4-6 MeV. The $\Theta^{+}$width also depends on the underlying assumptions of the background shape, but in all cases the uncertainties are large enough to be consistent with a width of $1 \mathrm{MeV}$. The ZEUS experiment [14] measured an intrinsic width of $8 \pm 4 \mathrm{MeV}$ for the $\Theta^{+}$ which is about two standard deviations away from a $1 \mathrm{MeV}$ width. It is worth mentioning that width quoted here is from a combination of $p K^{0}$ and $\bar{p} K^{0}$ data, and the ZEUS experiment concludes that the width is above, but consistent with, the experimental resolution of $2 \mathrm{MeV}$. 


\subsection{Theoretical Speculation}

If the $\Theta^{+}$width is really as narrow as $1 \mathrm{MeV}$, then both quark and soliton models will have a difficult time to explain such a narrow width. This fact alone is perhaps one of the most worrisome aspects to the question of $\Theta^{+}$existence. However, it is possible to explain the narrow width 28 if the $\Theta^{+}$is a mixture of two mass eigenstates. One suggestion [35] is that the diquark model of Jaffe and Wilczek 24] and the diquark-triquark model of Karliner and Lipkin 23] could mix. In this case, the mixing can conspire to decouple one of the eigenstates from $\mathrm{KN}$ decay, giving it a narrow width (and the other eigenstate with a wide width 28]). Such a mechanism would require fine-tuning of the mixing, which is not a desirable feature of any model, but at least allows for a possible explanation of the narrow width.

Another paradox is how the $\Theta^{+}$can be produced if the width is so narrow. For photoproduction, the most straight-forward calculation of the production mechanism is through the t-channel, where the width is related to the coupling constant for t-channel kaon exchange. If the width is about $1 \mathrm{MeV}$, then this diagram is suppressed. However, $K^{*}$ exchange is still possible, and this suggests experiments that could test this mechanism[28]. Another possibility is production through a cryptoexotic $N^{*}$ resonance 36. This was first suggested by the CLAS proton data [15]. If this is the dominant production mechanism, then some experiments with null results could be explained [36]. As enticing as these theoretical ideas may be, the ultimate test will be experiment.

\subsection{Experimental Outlook}

The best way to measure the width of the $\Theta^{+}$is with a high-resolution spectrometer, for a reaction that clearly identifies the strangeness in the final state. A proposal for the reaction $K^{+} p \rightarrow \pi^{+} \Theta^{+}$has been approved to run in 2005 at the KEK facility in Japan [37. The $K^{+}$beam at momentum $1.2 \mathrm{GeV} / \mathrm{c}$ will be incident on a liquid hydrogen target of length $10 \mathrm{~cm}$. The $\pi^{+}$particles will be detected in the SKS spectrometer with an expected resolution of about 1 $\mathrm{MeV}$. The cross section is estimated to be about $80 \mu \mathrm{b}$, which would provide about 3500 counts in the $\Theta^{+}$peak. If the cross section is up to four times lower than expected, the $\Theta^{+}$should still be visible above background with about 6 $\sigma$ statistical significance for a $\Theta^{+}$intrinsic width of $2 \mathrm{MeV}$ or less. This direct measurement avoids the difficulty of Fermi momentum and final state interactions that are always present for a deuterium target. If a clear peak is seen, this experiment will determine precise values for the $\Theta^{+}$mass and width.

\section{How can the parity be measured?}

The parity of the $\Theta^{+}$is closely related to questions of the $\Theta^{+}$structure. If the $\Theta^{+}$exists with a narrow width, then it likely has angular momentum $L>0$ otherwise it will just fall apart into a kaon and a nucleon. Also, it is known that the $L=0$ partial waves of the $K N$ system are repulsive 38 and hence do 
not show resonance structure. The same is true for the $L=1$ isovector partial wave, $P_{13}$, whereas the isoscalar $P_{11}$ is attractive. From this heuristic reasoning, the most likely parity assignment for the $\Theta^{+}$is positive parity with $J^{\pi}=\frac{1}{2}^{+}$. (Recall that the intrinsic parity of the $\bar{s}$ quark is negative.)

On the other hand, lattice gauge calculations suggest that the lowest mass eigenstate of the $u d u d \bar{s}$ system is just above the $K N$ threshold with negative parity [39, 40] and the next mass eigenstate (with positive parity) is several hundred $\mathrm{MeV}$ higher. However, these calculations are done in the quenched approximation with heavy quark masses, and further research is necessary to determine if chiral symmetry effects are significant when realistic quark masses are used 41. In fact, not all lattice calculations agree, as one that uses optimal domain-wall fermions 42 gives positive parity for the lowest mass eigenvalue. Another lattice calculations uses several different interpolating operators 43], some of which may have a larger overlap with the $\Theta^{+}$wave function than others, and the results are mixed: for some operators a state with negative parity is found but not for other interpolating fields. Although lattice calculations have made significant progress in past years 44 it is still too soon to get a conclusive prediction of the $\Theta^{+}$existence or parity from the lattice.

There are other indirect indications of the parity of the $\Theta^{+}$based on experiments. The cross sections that have been reported for photoproduction experiments are on the order of 50-100 nb. Calculations based on Regge theory 45, 46. 47. give cross sections in this range for positive parity, but are a factor of 10 or more lower for negative parity. In other words, if the $\Theta^{+}$is produced by a t-channel process, photoproduction experiments would not have the sensitivity to see a peak unless the parity is positive. Of course, it is possible that the $\Theta^{+}$ is produced by decay of an intermediate $N^{*}$ resonance in a s-channel process, which is not included in these calculations (because of the unknown $N^{*}$ coupling constant). If more experimental information on, say, the angular distribution of $\Theta^{+}$production can be measured, the assumptions in these Regge model calculations can be tested. Similarly, the COSY-TOF result gives a cross section of about $0.4 \mu \mathrm{b}$ which is comparable with calculations for positive parity 48 but is more than a factor of ten too large if the $\Theta^{+}$has negative parity.

\subsection{Experimental Outlook}

Clearly, a direct measurement of the $\Theta^{+}$parity is desired. For photoproduction experiments, the beam and target can be polarized, but even if the $\Theta^{+}$is produced in a state with known polarization, the parity of the $\Theta^{+}$can only be found with a measurement of the recoil polarization of its decay nucleon [49], which requires thousands of $\Theta^{+}$events and specialized detectors. However, the situation is much more favorable for hadronic beams, where the Pauli exclusion principle restricts the symmetry of the wave function. Near threshold, where only one or two partial waves are important, experiments with polarized beam and polarized target can be used to determine the $\Theta^{+}$parity. The idea is quite simple [49, 50]. For the reaction $p p \rightarrow \Sigma^{+} \Theta^{+}$when the beam and target spins are parallel, both isospin and spin components of the wave function are symmet- 
ric, allowing only odd angular momentum (negative parity) states. Similarly, antiparallel spins gives only positive parity states. Experimentally, the cross section for $\Theta^{+}$production near threshold (where the $\Sigma^{+}$and $\Theta^{+}$are in a relative s-wave or p-wave) is much bigger in one spin allignment than the other. Such measurements are planned after 2007 at COSY.

\section{$5 \quad$ Summary and Conclusions}

With both positive and null evidence for the $\Theta^{+}$from a variety of experiments, it is difficult to conclude whether the $\Theta^{+}$exists or not. Furthermore, the theoretical difficulties to explain a possible narrow width, perhaps as small as $1 \mathrm{MeV}$, suggest that if the $\Theta^{+}$exists, it is very unusual indeed. Nor is guidance from lattice gauge theory helpful, as some calculations show evidence for a negative parity resonance, one gets positive parity, and others do not see a resonance in either parity. So the job of proving or disproving the $\Theta^{+}$existence is currently an experimental task.

The question of existence of the $\Theta^{+}$will not be solved by a "scorecard" approach. With ten experiments with statistically-limited positive evidence and almost as many experiments with null evidence (with higher statistics but also uncertain backgrounds) the question is how to do better experiments. Several new experiments are on the horizon, and so the existence question should be answered within a year or so. The high-statistics experiments on hydrogen and deuterium using the CLAS detector have just finished, and the analysis of these data are proceeding rapidly. A new experiment with a $K^{+}$beam on a hydrogen target has been scheduled for 2005, and calculations predict a $\Theta^{+}$peak with hundreds of counts and few-MeV resolution. The COSY facility has also scheduled another run for the TOF detector, which could more than double their statistics. These are just a few examples of the world-wide efforts to determine if the $\Theta^{+}$exists.

\section{Acknowledgments}

I am grateful to my colleagues in the LEPS collaboration (Japan) and the CLAS collaboration (USA) who have contributed heavily to this area. I thank Harry Lipkin, Marek Karliner, Bob Jaffe, Ted Barnes, Takashi Nakano and colleagues from CLAS for helpful comments. This work was supported in part by the National Science Foundation and the Research Center for Nuclear Physics in Osaka, Japan.

\section{References}

[1] See references 5-8 and 12-18 below.

[2] See reference 19 below. 
[3] S. Olsen, MESON 2004 conference; F. Harris, ibid.

[4] See references 36-39 below.

[5] T. Nakano et al. (the LEPS collaboration), Phys. Rev. Lett. 91, 012002 (2003).

[6] V.V. Barmin et al., Phys. Atom. Nuclei 66, 1715 (2003).

[7] S. Stepanyan et al. (the CLAS collaboration), Phys. Rev. Lett. 91, 25001 (2003).

[8] J. Barth et al., Phys. Lett. B572, 127 (2003).

[9] A. Dzierba, et al., Phys. Rev. D69 (2004) 051901.

[10] K. Hicks, Hadron 2003 conference proceedings, AIP Publicaitons, 2003.

[11] Michael Ostrick, Pentaquark 2003 workshop, Jefferson Lab, November 2003, http://www.jlab.org/intralab/calendar/archive03/pentaquark/

[12] A.E. Asratyan, A.G. Dolgolkenko and M.A. Kubantsev, Phys. Atom. Nucl. (2004); hep-ex/0309042

[13] A. Airapetian et al. (HERMES), Phys. Lett. B585 (2004) 213; hep-ex/0312044

[14] ZEUS collaboration, Phys. Lett. B591 (2004) 7-22; hep-ex/0403051.

[15] V. Kubarovsky et al., Phys. Rev. Lett. 92, 032001 (2004).

[16] D. Weygand, MESON 2004 conference proceedings.

[17] K. Killian, MESON 2004 conference proceedings.

[18] T. Nakano, NSTAR 2004 conference website.

[19] http://www.qnp2004.org

[20] R. De Vita, NSTAR 2004 proccedings, in press.

[21] COSY-TOF collaboration, nucl-ex/0403012

[22] S. Capstick, P. Page and W. Roberts, Phys. Lett. B570 (2003) 185-190.

[23] M. Karliner and H.J. Lipkin, Phys. Lett. B575 (2003) 249; hep-ph/0307243.

[24] R. Jaffe and F. Wilczek, Phys. Rev. Lett. 91, 232003 (2003).

[25] D. Diakonov, V. Petrov and M. Polyakov, Zeit. Phys. A 359, 305 (1997).

[26] D. Diakonov, V. Petrov and M. Polyakov, hep-ph/0404212 
[27] J.R. Ellis, M. Karliner and M. Praszalowicz, JHEP 0405:002 (2004); hep-ph/0401127

[28] M. Karliner and H.J. Lipkin, Phys. Lett. B586 (2004) 393.

[29] S. Nussinov, hep-ph/0307357

[30] R.N. Cahn and G.H. Trilling, Phys. Rev. D69, 011501 (2004).

[31] R.A. Arndt, I.I. Strakovsky and R.L. Workman, Phys. Rev. C68, 042201(R) (2003).

[32] A. Sibirtsev, J. Haidenbauer, S. Krewald and Ulf-G. Meissner, hep-ph/0405099

[33] W.R. Gibbs, nucl-th/0405024.

[34] T. Barnes, private communication.

[35] B. Jennings and K. Maltman, Phys.Rev. D69 (2004) 094020.

[36] M. Karliner and H.J. Lipkin, hep-ph/0405002

[37] K. Imai et al., proposal to KEK (2004).

[38] J.S. Hyslop, R.A. Arndt, L.D. Roper, R.L. Workman, Phys. Rev. D 46, 961 (1992).

[39] F. Csikor, Z. Fodor, S.D. Katz and T.G. Kovacs, Jour. High Energy Physics 11, 70 (2003); hep-lat/0309090

[40] S. Sasaki, hep-lat/0310014

[41] F.X. Lee, MESON 2004 conference; N. Mathur et al., hep-ph/0406196

[42] Ting-Wai Chiu and Tung-Han Hsieh, hep-ph/0403020

[43] J. Negele, GDH2004 conference website.

[44] C. Morningstar, CIPANP conference, NY (2003).

[45] Y. Oh, H. Kim, S.H. Lee, hep-ph/0311054

[46] W. Liu, C.M. Ko, nucl-th/0309023

[47] T. Hyodo, A. Hosaka, E. Oset, nucl-th/0307105

[48] S.I. Nam, A. Hosaka, H.-Ch. Kim, hep-ph/0308313

[49] A.W. Thomas, K. Hicks and A. Hosaka, Prog. Theor. Phys. 111, 291 (2004).

[50] Ulf-G. Meissner, MESON 2004; C. Hanhart et al., Phys. Lett. B 590 (2004) 39. 